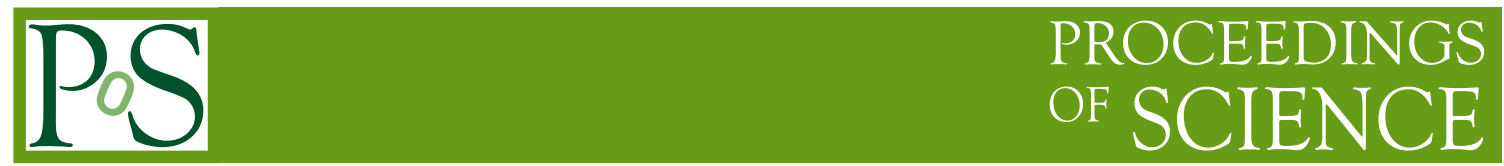

\title{
Studies of the Beam backgrounds at the CEPC
}

\author{
Haoyu Shi, ${ }^{a, *}$ Hongbu Zhu, ${ }^{a}$ Wei Xu, ${ }^{a, b}$ Sha Bai ${ }^{a}$ and Xinchou Lou ${ }^{a, b}$ \\ ${ }^{a}$ Institute of High Energy Physics, CAS \\ $19 b$ Yuquan Road, Beijing, China \\ ${ }^{b}$ University of Chinese Academy of Sciences \\ 19a Yuquan Road, Beijing, China
}

E-mail: shihy@ihep.ac.cn

The Circular Electron Positron Collider (CEPC) has been proposed for the Higgs and electroweak measurements with unprecedented precision. To achieve the required performance precision, it is necessary to understand, characterize and then mitigate the beam backgrounds from different sources that are critical for the machine and detector design and operation. In this paper, preliminary results will be presented for the beam backgrounds originating from the off energy beam particles. With the machine design presented in the CEPC Conceptual Design Report (CDR), detailed simulation including generation of the off energy beam particles originating from different processes and tracking of the particles has been performed. Four sets of collimators are introduced to prevent such off energy beam particles from re-entering the interaction region and reduce the background level.

40th International Conference on High Energy physics - ICHEP2020

July 28 - August 6, 2020

Prague, Czech Republic (virtual meeting)

${ }^{*}$ Speaker 


\section{Introduction}

Circular Electron Position Collider (CEPC) is a Higgs factory proposed by the Chinese high energy physics community and aimed to measure the properties of the Higgs boson and electroweak parameters with unprecedented precision. It will be a $100 \mathrm{~km}$ double ring machine with a crossing angle of 33 mrad. Its main design machine parameters from the Conceptual Design Report $(\mathrm{CDR})[1,2]$ are listed in Table 1 . To achieve optimal performance of the machine and detectors and ultimately to realize the precision physics program, it is critical to understand thoroughly the complex beam backgrounds originating from different sources, including the two main types, photons and off energy beam particles. In this paper, we report the preliminary results of the off beam energy beam particles.

Table 1: CEPC main machine parameters as presented in CDR.

\begin{tabular}{cccc}
\hline Operation Mode & Higgs $(240 \mathrm{GeV})$ & $\mathrm{W}(160 \mathrm{GeV})$ & $\mathrm{Z}(91 \mathrm{GeV})$ \\
\hline Particles/bunch $N_{e}\left[10^{10}\right]$ & 15 & 12 & 8 \\
Bunch Number & 242 & 1524 & 12000 \\
Horizontal beam size $\sigma_{x}[\mu \mathrm{m}]$ & 20.9 & 13.9 & 6.0 \\
Vertical beam size $\sigma_{y}[\mu \mathrm{m}]$ & 0.06 & 0.049 & 0.078 \\
Energy spread[\%] & 0.134 & 0.098 & 0.080 \\
$\mathscr{L}\left[10^{34} \mathrm{~cm}^{-2} \mathrm{~s}^{-1}\right]$ & 3 & 10 & 17 \\
\hline
\end{tabular}

\section{Off Energy Beam Particles}

Table 2: Beam loss processes and corresponding beam lifetimes.

\begin{tabular}{ccc}
\hline Process & Beam Lifetime & Comments \\
\hline Touschek Effect & $>1000 \mathrm{~h}$ & \\
Beam Gas Comlomb Scattering & $>400 \mathrm{~h}$ & Residual Gas CO, $10^{-7} \mathrm{~Pa}$ \\
Beam Gas Bremsstrahlung Scattering & $63.8 \mathrm{~h}$ & Residual Gas CO, $10^{-7} \mathrm{~Pa}$ \\
Beam Thermal Photon Scattering & $50.7 \mathrm{~h}$ & \\
Radiative Bhabha Scattering & $74 \mathrm{~min}$ & \\
Beamstrahlung & $80 \mathrm{~min}$ & \\
\hline
\end{tabular}

Beam particles can lose energy through several scattering processes, including beamstrahlung, radiative Bhabha scattering, beam thermal photon scattering, beam gas scattering and Touschek scattering. If the energy loss exceeds the energy acceptance of $1.5 \%$ as designed for CEPC CDR, the beam particle will get lost and hit the beam pipe. Off energy beam particles lost in the interaction region will interact with the machine and detector elements, and can introduce unwanted radiation backgrounds and impair the detector performance. Therefore, they must be suppressed with appropriate measures. The scattering processes expected at the CEPC and their corresponding beam lifetimes are listed in Table 2. Shorter lifetime indicates higher impacts of the beam loss process under investigation. Given their relative importance, beamstrahlung(BS)[3-6], 
radiative Bhabha scattering(RBB)[5], beam thermal photon scattering $(\mathrm{BTH})[5,7]$ and beam gas bremsstrahlung scattering(BGS)[5, 6] have been studied in detail and corresponding results are discussed below.

\section{Event Simulation}

The off energy beam particle simulation process consists of three main steps: event generation, particle tracking and detector simulation. Each type of scattering process is first generated with our customized Monte Carlo generator[5] and cross-checked with other widely used generators such as BBBrem[8] to produce consistent results. BTH and BGS scattering events are simulated in the interaction region and up to $200 \mathrm{~m}$ upstream of the interaction point. The resulting energy distributions of the scattered particles from multiple scattering processes are shown in Figure 1.

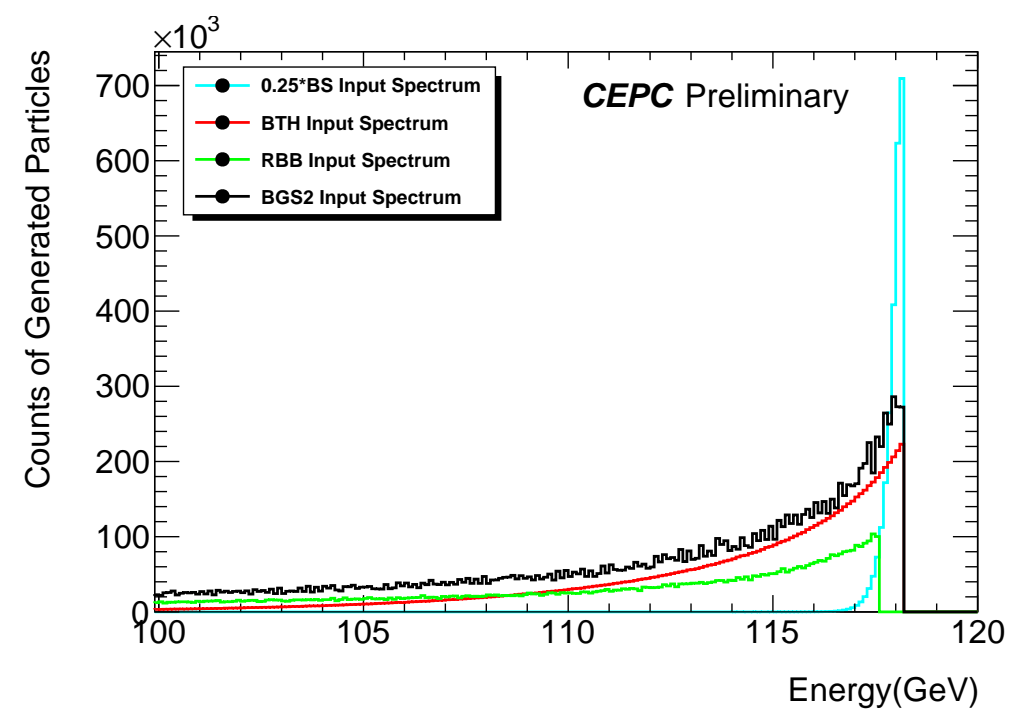

Figure 1: Energy distributions of the off energy beam particles from different scattering processes.

Generated particles are further tracked with the Strategic Accelerator Design(SAD)[9] and propagated into the interaction region. Accelerator components are sliced into small sections of $10 \mathrm{~cm}$ in length to improve the simulation accuracy. During the tracking process, if a beam particle travels beyond the beam pipe aperture, this particle will be marked as lost. At the same time, the particle position, momentum and other relevant information are recorded and will be used as the input into the detector simulation at the next stage. As shown in Figure 2(a), BS off energy beam particle loss happens predominately to the particles traveling along the ring after multiple turns but eventually kicked off the orbit when re-entering the interaction region. Beam particle loss after multiple turns also make considerable contributions in the other three processes. Figure 2(b), (c) and (d) show the results from the BGS, BTH and RBB, respectively. In particular, "Oth-turn" represents the scattered particles lost even before reaching the interaction point. Unfortunately, the off energy beam particles from the "0th-turn" and also the "1st-turn" downstream due to the large angle change and/or the large energy loss, are nearly impossible to be mitigated. But for the off energy beam particles from the "1st-turn" upstream and the multiple turns can and must be mitigated with properly designed collimators and other measures. 


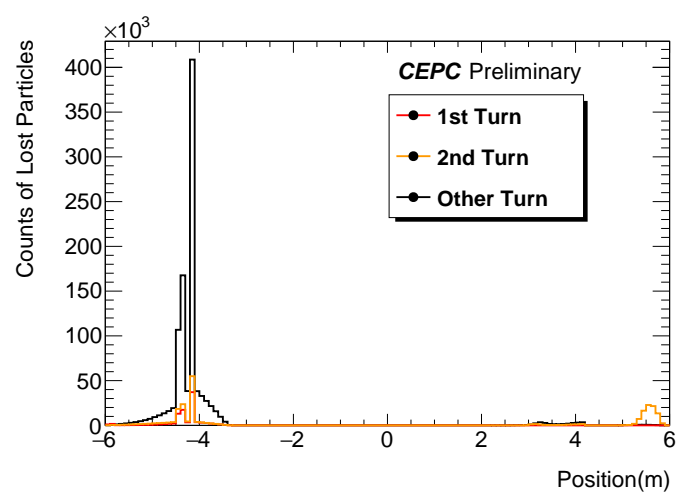

(a) BS

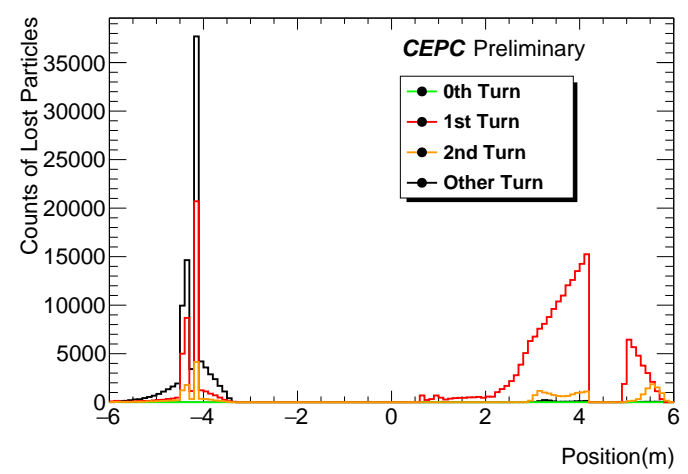

(c) $\mathrm{BTH}$

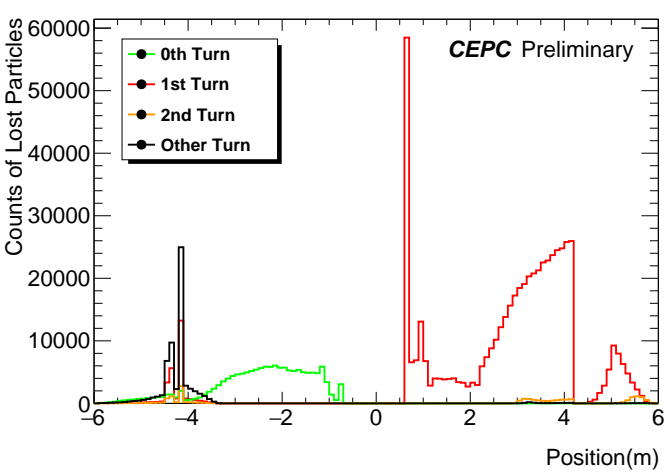

(b) BGS

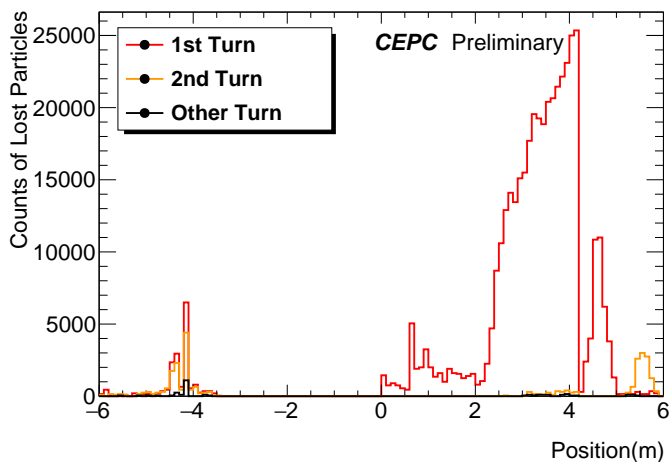

(d) RBB

Figure 2: Lost distributions of the off energy beam particles from BS, BGS, BTH and RBB, respectively.

\section{Collimator Design and Effectiveness}

Table 3: Design parameters of the four sets of colliamators.

\begin{tabular}{ccccc}
\hline Name & Position & Distance to IP & Beta Function & Range of Half width allowed \\
\hline APTX.1 & D1I.1897 & $2139.06 \mathrm{~m}$ & $113.83 \mathrm{~m}$ & $2.2 \sim 9.68 \mathrm{~mm}$ \\
APTX.2 & D1I.1894 & $2208.63 \mathrm{~m}$ & $113.83 \mathrm{~m}$ & $2.2 \sim 9.68 \mathrm{~mm}$ \\
APTX.3 & D1O.10 & $1832.52 \mathrm{~m}$ & $113.83 \mathrm{~m}$ & $2.2 \sim 9.68 \mathrm{~mm}$ \\
APTX.4 & D1O.14 & $1901.09 \mathrm{~m}$ & $113.83 \mathrm{~m}$ & $2.2 \sim 9.68 \mathrm{~mm}$ \\
\hline
\end{tabular}

Collimators can be inserted into the beam line to reduce the number of particles lost in the IR. The aperture size of the collimators should be as small as possible to block the off energy beam particles but still large enough not to disturb the beam. In the CEPC CDR design, four sets of collimators are introduced in the positron ring and placed only in the horizontal planes. Two of them are located upstream of the interaction point, and the other two are located downstream. Their distances to the interaction point range from 1800 meters to 2300 meters. Table 3 shows the design parameters of the collimators. The collimator half widths are set to $5 \mathrm{~mm}\left(\sim 13 \sigma_{x}\right)$ in the horizontal plane and should not affect the beam quantum lifetime. Figure 3 shows the significant reduction 


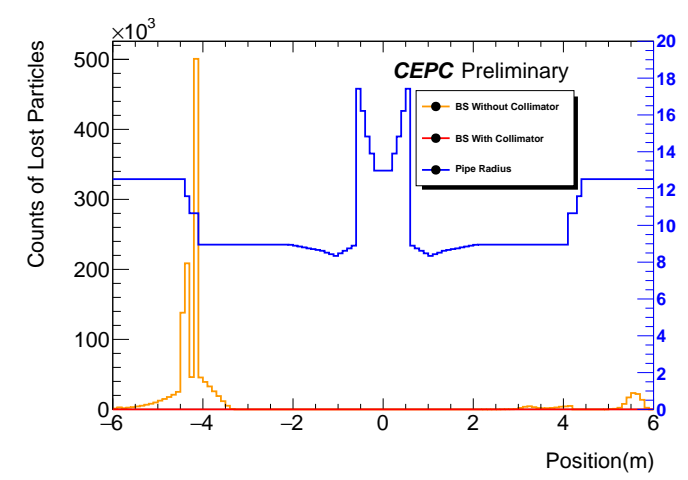

(a) BS

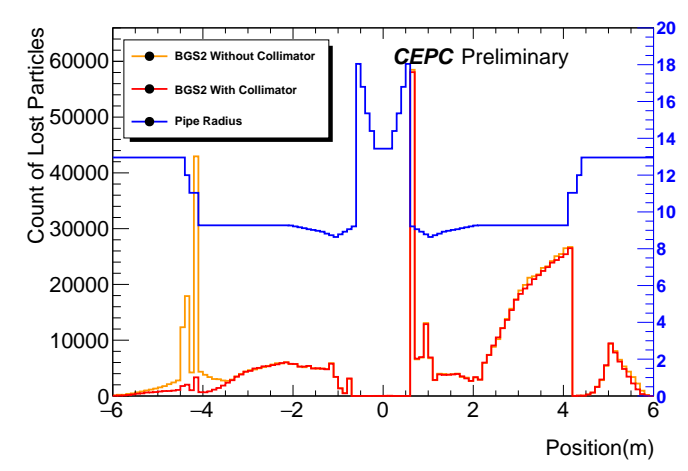

(c) BGS

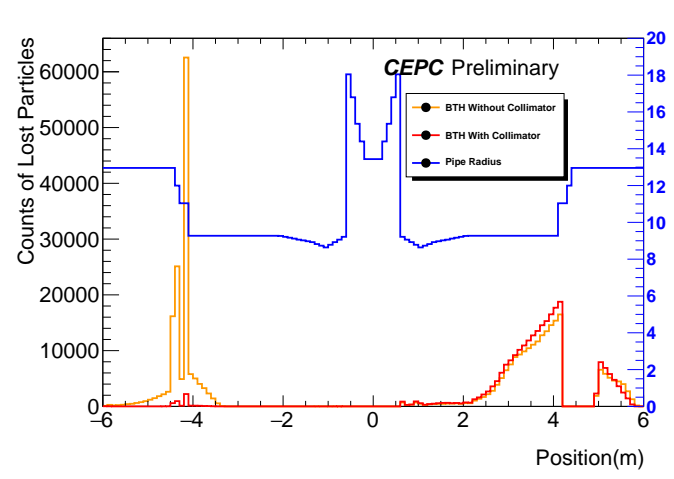

(b) $\mathrm{BTH}$

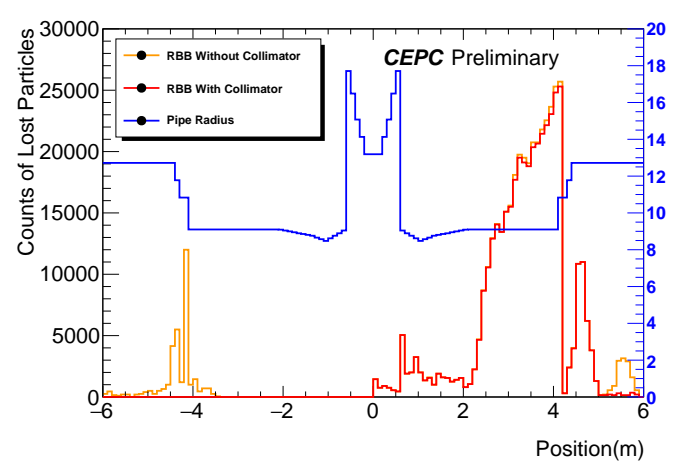

(d) RBB

Figure 3: Off energy beam particle loss distribution for the BS, BGS, BTH and RBB with and without collimators, respectively.

of the off energy beam particle loss for each process before and after introducing the collimators, in particular those from the multi-turn upstream loss. And Figure 4(b) shows the combined loss rates from the four processes and indicate the main remaining background contribution from the downstream loss.

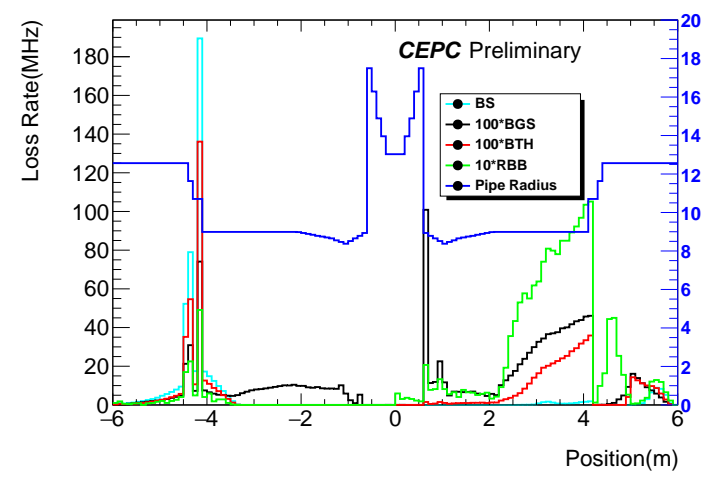

(a) Without collimators

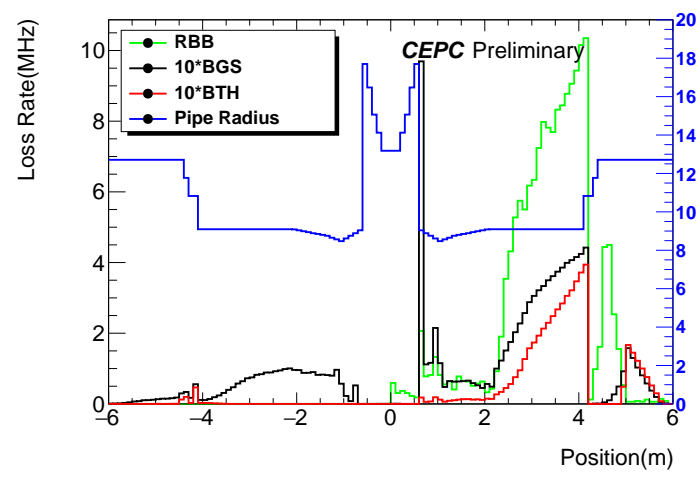

(b) With collimators

Figure 4: The lost distribution rates of the off energy beam particles with and without the collimators. 


\section{Conclusion}

CEPC has been proposed as a Higgs factory aimed to measure the properties of the Higgs boson with unprecedented precision. Understanding and controlling the beam backgrounds will be essential to achieve the optimal machine and detector performance. Detailed investigation of the off energy beam particle background has been carried out. The simulation includes event generation, particle tracking and detector simulation. Preliminary results are obtained from the particle tracking and four sets of collimators have been introduced to suppress effectively the resulting backgrounds. Further studies will be performed to compute the resulting background levels in the detector and extended to cover backgrounds originating from other sources.

\section{Acknowledgement}

This project is jointly supported by the CAS Center for Excellence in Particle Physics (CCEPP) and the Beijing Municipal Science \& Technology Commission Project (Z181100004218003 and Z191100007219010).

\section{References}

[1] C.S. Group et al., Cepc conceptual design report, arXiv preprint arXiv:1809.00285 (2018) .

[2] C.S. Group et al., Cepc conceptual design report: Volume 2-physics \& detector, arXiv preprint arXiv:1811.10545 (2018) .

[3] D. Schulte, Study of electromagnetic and hadronic background in the interaction region of the tesla collider, Tech. Rep. (1997).

[4] Q.-L. Xiu, H.-B. Zhu, T. Yue and X.-C. Lou, Study of beamstrahlung effects at cepc, Chinese Physics C 40 (2016) 053001.

[5] T. Yue, The Study of Beam-induced Background of Electron-Positron Colliders, Ph.D. thesis, 2016.

[6] P. Lewis, I. Jaegle, H. Nakayama, A. Aloisio, F. Ameli, M. Barrett et al., First measurements of beam backgrounds at superkekb, Nuclear Instruments and Methods in Physics Research Section A: Accelerators, Spectrometers, Detectors and Associated Equipment 914 (2019) 69.

[7] H. Burkhardt, Monte carlo simulation of scattering of beam particles and thermal photons, Tech. Rep. CERN-SL-Note-93-73-OP (1993).

[8] R. Kleiss and H. Burkhardt, Bbbremmonte carlo simulation of radiative bhabha scattering in the very forward direction, Computer physics communications 81 (1994) 372.

[9] K. Hirata, An introduction to sad (strategic accelerator design), in Second Advanced ICFA Beam Dynamics Workshop, CERN, pp. 88-04, 1988. 